\title{
A New Edge Detection Method based on Max and Min Values of the Pixels
}

\author{
Muthanna Ali Saihood ${ }^{1}$, G. Prathibha ${ }^{2}$ \\ ${ }^{1}$ Research Scholar, PG, University College of Engineering \& Technology, Acharya Nagarjuna University, A.P., India \\ Guntur, Andhra Pradesh, India \\ ${ }^{2}$ Assistant Professor, Department of ECE, University College of Engineering \& Technology, Acharya Nagarjuna University \\ Guntur, Andhra Pradesh, India
}

\begin{abstract}
Edge is defined as boundary between two different intensities of the image pixels which means difference in intensity of the pixel in the image. The edges for the image are always the important features that introduce an indication for a higher frequency. There are many methods of programs in mat lab to perform edge detection. The most common method for edge detection is to calculate the differentiation of an image called Prewitt edge. This paper proposes a method to detect an edge of image depending on the maximum and minimum of the pixel values that requires less computation time than Prewitt and Sobel Operators.
\end{abstract}

Keywords: Image process, Edge detection, Pixels, Sobel Operators.

\section{Introduction}

Edge detection techniques transform images to edges benefiting from the changes of grey intensity of the images. Edges are the sign of lack of continuity and ending. As a result of this transformation, edge image is obtained without encountering any changes in physical qualities of the main image, the edge in the image is a significant local change in the image intensity, usually associated with the discontinuity in either the image intensity or the first derivative of the image intensity. Discontinuities in the image intensity can be either step edges, where the image intensity suddenly changes from one value on one side of the discontinuity to a different value on the opposite. Detection includes three steps that are Filtering, Enhancement and Detection. Image is often corrupted by random variations in intensity values, that is called the noise. Some common types of noise is Gaussian noise, the filtering process is done to reduce the noise that produce in a loss of edge strength. In order to facilitate the detection of edges, it is basic to determine the changes in intensity of the neighbourhood of the points. Different methods of edge detection may be grouped into two parts:

- Gradient based Edge Detection: The gradient method detects the edges by looking for the maximum and minimum in the first derivative of the image.

- Laplacian based Edge Detection: The Laplacian method searches for zero crossings in the second derivative of the image to find edges. An edge has the onedimensional shape of a ramp and calculating the derivative of the image can highlight its location.

Four most frequently used edge detection Operators are used for comparison. These are Roberts Operator, Sobel Operator, Prewitt Operator, Canny Operator.

\section{The Proposed Method}

This method detects the edge of the image using a mask which is a sub matrix with four elements or pixels. The mask scans all the image pixels by shifting horizontally and vertically on the image, and every scan position gets the maximum value and minimum value of the pixels and after that find the difference between the maximum value and minimum value of the pixels to get value of the factor $a_{4}$. Compare the value of $a_{4}$ with several numbers to get the best edge of the image, at first compare the value of $a_{4}$ with 100 if the value is greater or equal than 100 this mean the location of mask on edge is in the first column values of the two elements or pixels of the mask 0 or black color to recognize the image edge, else if the value of the $a_{4}$ is less than 100 means the mask location is not image edge and assign all values of the four elements or pixels of the mask 255 or white color to recognize the background of the image. It can be seen that if the value of $\mathrm{a}_{4}$ with number less than 100 for example 50 then we can increase the accuracy of the image edge. Figure 1 explains the edge of image along with change in the intensity values and position of the pixels.

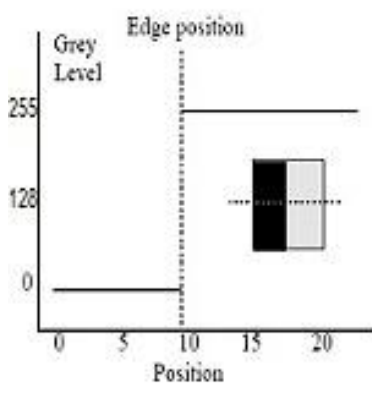

(a)

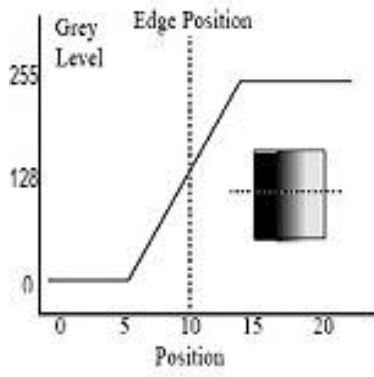

(c)

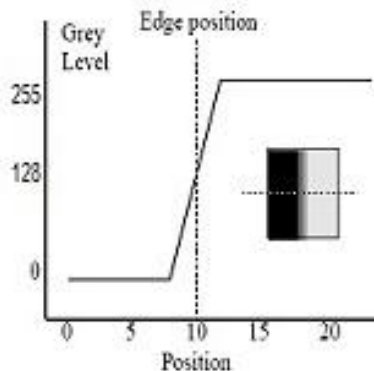

(b)

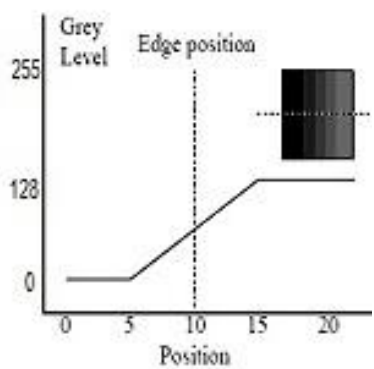

(d) 


\section{International Journal of Science and Research (IJSR) \\ ISSN (Online): 2319-7064 \\ Index Copernicus Value (2013): 6.14 | Impact Factor (2014): 5.611}

Figure 1: Various edges.(a) The change in level occurs suddenly exactly at pixel 10. (b) Ramp edge. (c) More gradation edge centered at pixel 10. (d) The most gradation edge centered at pixel 10, a smaller change with values of pixels.

Figure 1(a) change in the values of pixels occurs suddenly and the edge exactly at pixel with position 10 (very high clear edge). Figure 1 (b) Shows the change in the values of pixels occurs with low gradually and the edge between the pixels with position 8 and 12 (high clear edge). Figure 2 (c) Shows the change in the values of pixels occurs with low gradually and the edge between the pixels with position 6 and 14 (low clear edge). Figure 1 (d) Shows that a smaller change in the values of pixels occurs gradually and the edge is in between the pixels with position 5 and 15 (the edge is not clear).

The sub matrix mask contain for each state in the figure 1 is shown in the figures 2(a), 2(b), 2(c) and 2(d). The best value of $\mathrm{a}_{4}$ to detect an edge in figure 1(a) is shown in figure 2(a).

\begin{tabular}{|c|c|}
\hline 0 & 255 \\
\hline 0 & 255 \\
\hline
\end{tabular}

Figure 2(a): The sub matrix mask with values $(0,255)$

For this state, choose the value of $a_{4}$ is very high to detect the edge with high accuracy because the value of $\mathrm{a}_{4}$ is difference between the Max $\left(a_{2}\right)$ and Min $\left(a_{3}\right)$ for the sub array mask $a_{4}$ $=\mathrm{a}_{2}-\mathrm{a}_{3}$, for this state the $\operatorname{Max}\left(\mathrm{a}_{2}\right)=255$ and the Min $\left(\mathrm{a}_{3}\right)=$ 0 , then the value of factor $a_{4}=255-0, a_{4}=255$. Then for this image when we compare $\mathrm{a}_{4}$ with 255 then we can detect the edge perfectly. The sub matrix mask corresponding to the state in figure $1(\mathrm{~b})$ is shown in figure $2(\mathrm{~b})$.

\begin{tabular}{|l|l|}
\hline 50 & 200 \\
\hline 50 & 200 \\
\hline
\end{tabular}

Figure 2(b) : The sub matrix mask with values $(50,200)$

For this state, choose the value of $\mathrm{a}_{4}$ is high to detect the edge with high accuracy because the value of $a_{4}$ is difference between the Max $\left(a_{2}\right)$ and Min $\left(a_{3}\right)$ for the sub array mask $a_{4}$ $=a_{2}-a_{3}$, for this state the Max $\left(a_{2}\right)=200$ and the Min $\left(a_{3}\right)=$ 50 , then the value of factor $\mathrm{a}_{4}=200-50, \mathrm{a}_{4}=150$. Then to detect the edge of this image perfectly compare $\mathrm{a}_{4}$ with 150 . The third state that corresponding to Figure-1(c) and the sub matrix mask at this position as shown in Figure-2(c)

\begin{tabular}{|c|c|}
\hline 80 & 130 \\
\hline 80 & 130 \\
\hline
\end{tabular}

Figure 2(c): The sub matrix mask with values $(80,130)$
For this state, choose value of $\mathrm{a}_{4}$ low to detect the edge with high accuracy because the value of $\mathrm{a}_{4}$ is difference between the $\operatorname{Max}\left(a_{2}\right)$ and $\operatorname{Min}\left(a_{3}\right)$ for the sub array mask $a_{4}=a_{2}-a_{3}$, for this state the $\operatorname{Max}\left(a_{2}\right)=130$ and the $\operatorname{Min}\left(a_{3}\right)=80$, then the value of factor $a_{4}=130-80, a_{4}=50$. Then to detect the edge of this image perfectly compare $a_{4}$ with 50 . The forth state that corresponding to Figure $1(\mathrm{~d})$ and the sub matrix mask at this position as shown in Figure 2(d)

\begin{tabular}{|c|c|}
\hline 90 & 110 \\
\hline 90 & 110 \\
\hline
\end{tabular}

Figure 2(d): The sub matrix mask with values $(90,110)$

For this state, choose the value of $\mathrm{a}_{4}$ is very low to detect the edge with high accuracy because the value of $a_{4}$ is difference between the Max $\left(a_{2}\right)$ and Min $\left(a_{3}\right)$ for the sub array mask $a_{4}$ $=\mathrm{a}_{2}-\mathrm{a}_{3}$, for this state the Max $\left(\mathrm{a}_{2}\right)=110$ and the Min $\left(\mathrm{a}_{3}\right)$ $=90$, then the value of factor $a_{4}=110-90, a_{4}=20$. Then to detect the edge of this image perfectly compare $a_{4}$ with 20 .

\section{Flow Chart}

The flowchart of the proposed method is shown in Figure 3 illustrating the edge detection process.

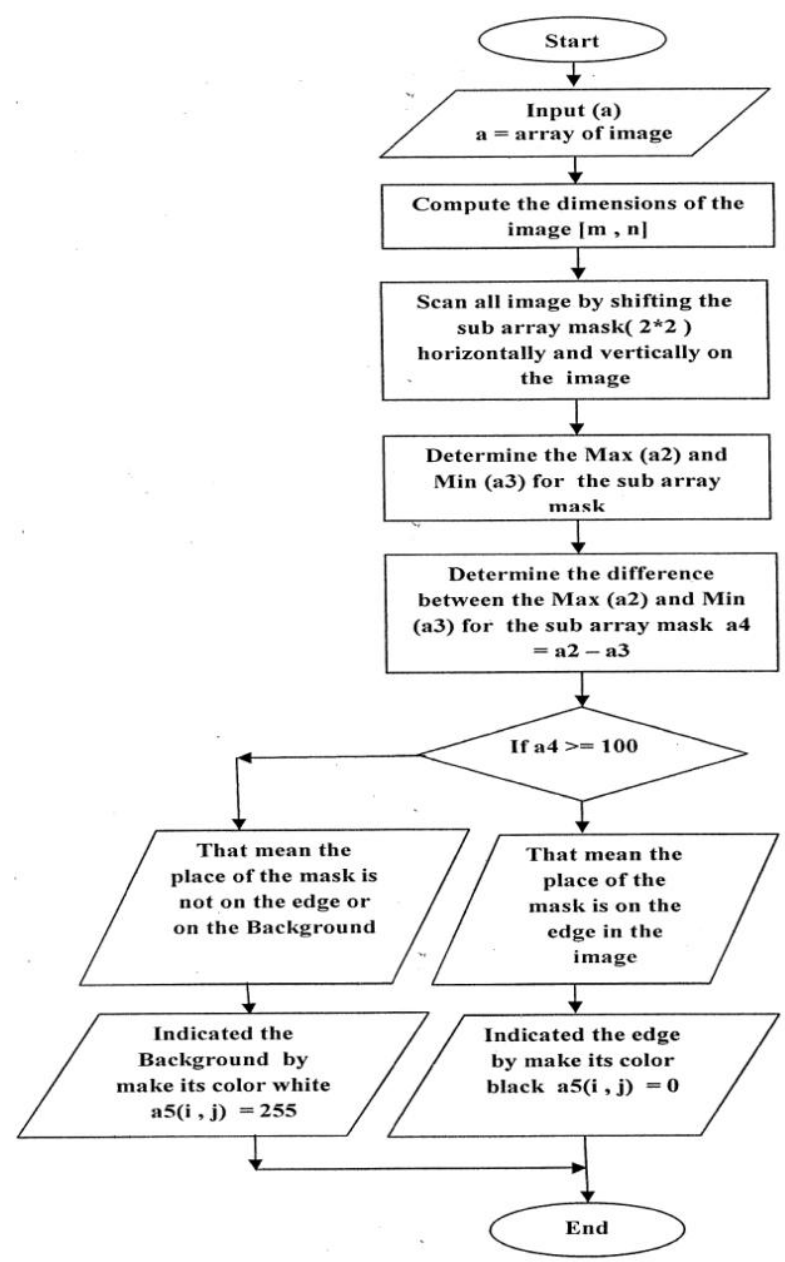

Figure 3: The flowchart of the proposed method

\section{Volume 4 Issue 12, December 2015}




\section{International Journal of Science and Research (IJSR) \\ ISSN (Online): 2319-7064}

Index Copernicus Value (2013): 6.14 | Impact Factor (2014): 5.611

\section{Results}

The proposed Max and Min Operator method is experimented on the standard Cameraman image is shown in Figure 4. The edge detection using Prewitt Operator applied to the standard Cameraman image is shown in Figure 5, which depicts that the edge is not continuous.

\section{ORIGINAL IMAGE}

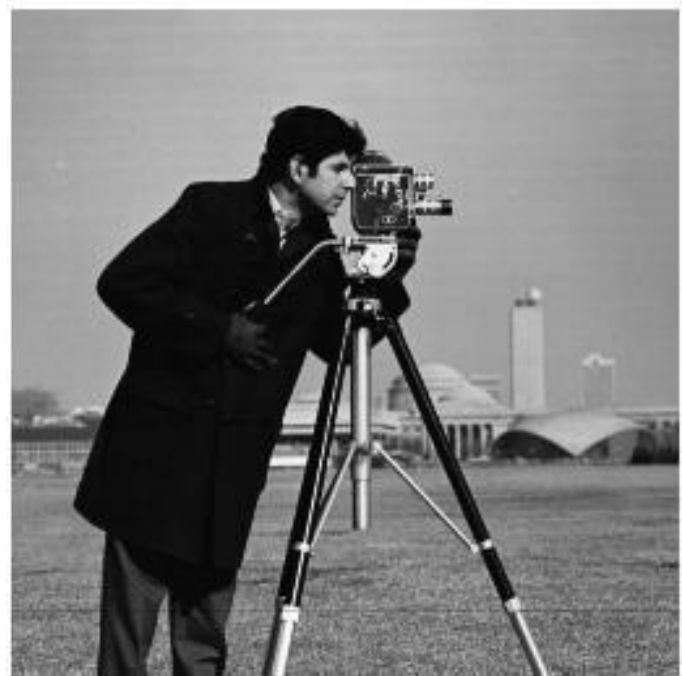

Figure 4: Cameraman image (Original)

\section{PERVERTEDGE IMAGE}

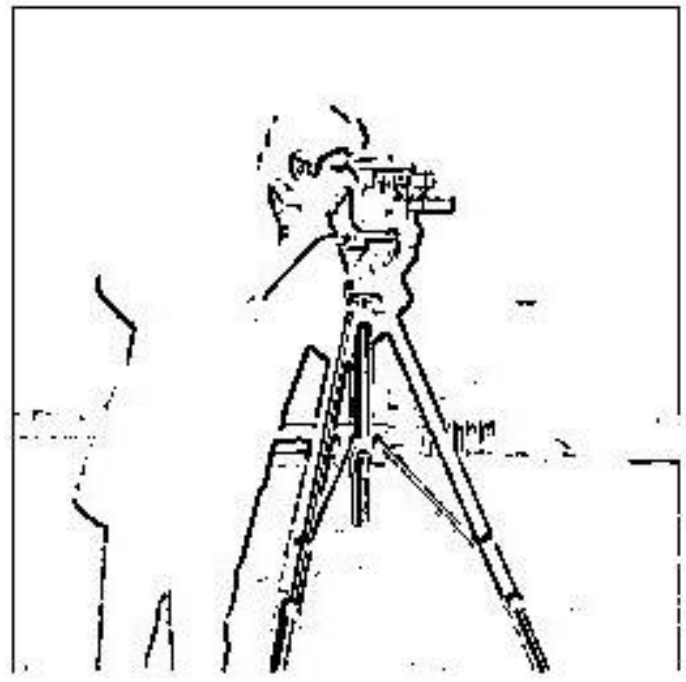

Figure 5: Edge of Pervertedge operator

So in order to obtain a perfect edge proposed method is applied with following different values of $\mathrm{a}_{4}: 100,50,30$ and 20 is shown in the figures 6(a), 6(b), 6(c) and 6(d) respectively.
EDGE FOR PROPOSED METHOD(a4>=100)

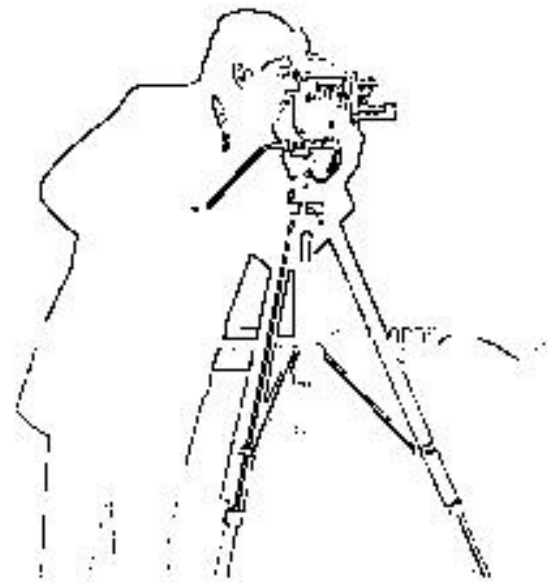

Figure 6(a): Edge for proposed $\operatorname{method}\left(\mathrm{a}_{4}>=100\right)$ EDGE FOR PROPOSED METHOD(a $4>=50)$

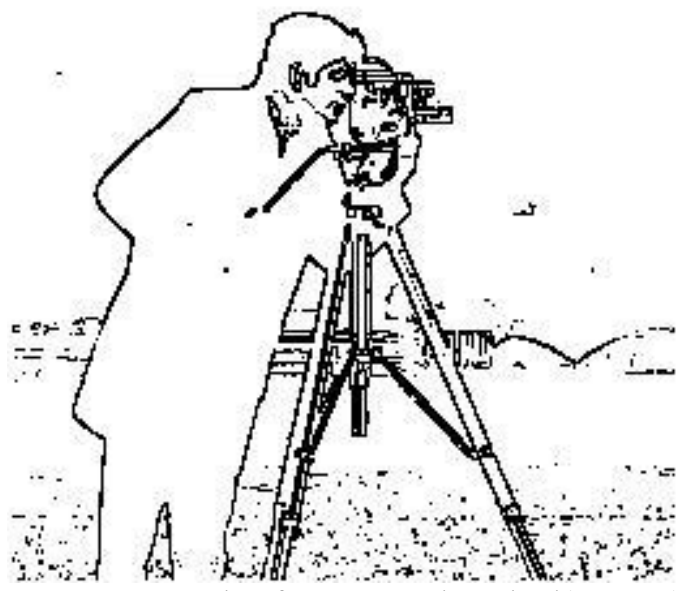

Figure 6(b): Edge for proposed method $\left(a_{4}>=50\right)$ EDGE FOR PROPOSED METHOD(a4>=30)

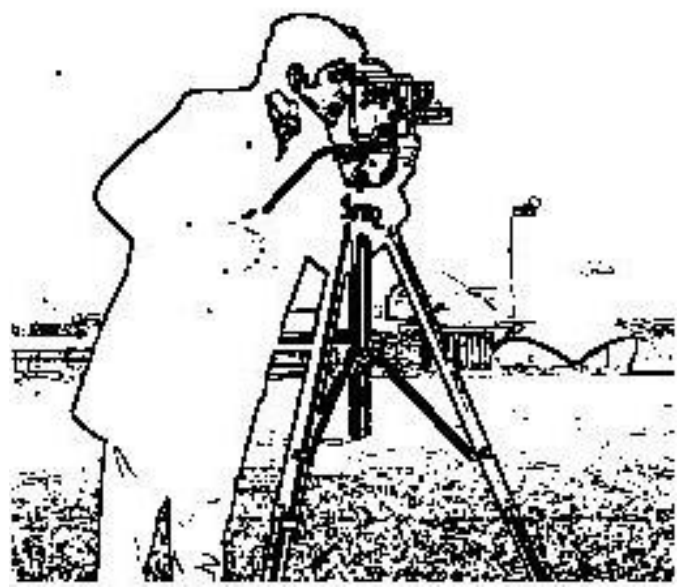

Figure 6(c): Edge for proposed method $\left(a_{4}>=30\right)$ 


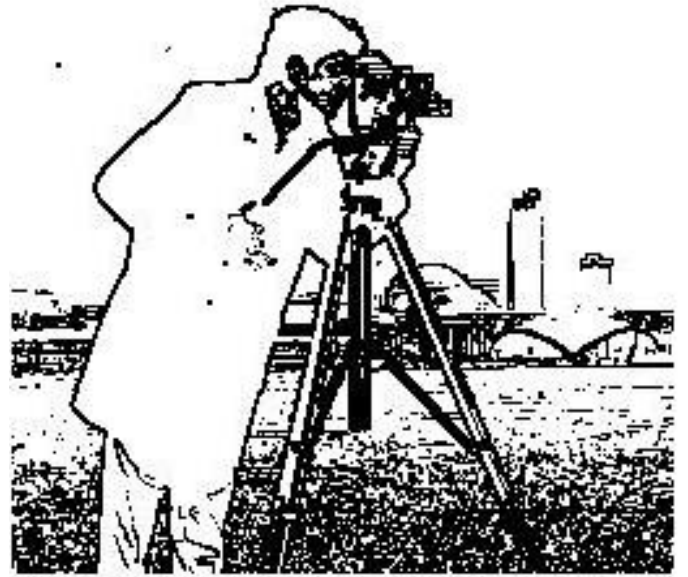

Figure 6(d): Edge for proposed method $\left(a_{4}>=20\right)$

Figures 6(a) and 6(b) illustrates the results of detected image when $a_{4}>=100$ and $a_{4}>=50$ respectively, but when need to detected more details of an image values of $a_{4}>=30$ and $a_{4}$ $>=20$ as shown in figures $6(\mathrm{c})$ and $6(\mathrm{~d})$ respectively. Figure 7 shows the original image of Lena. The results for Lena image by proposed method with values of $\mathrm{a}_{4}$ as 100,80 and 50 is shown in figures $8(\mathrm{a}), 8(\mathrm{~b})$ and $8(\mathrm{c})$ respectively.

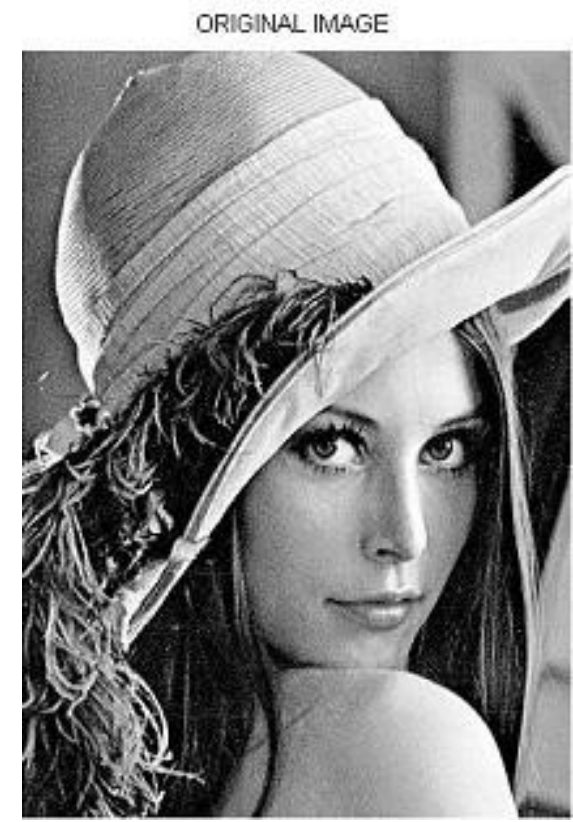

Figure 7: Lena image (Original)

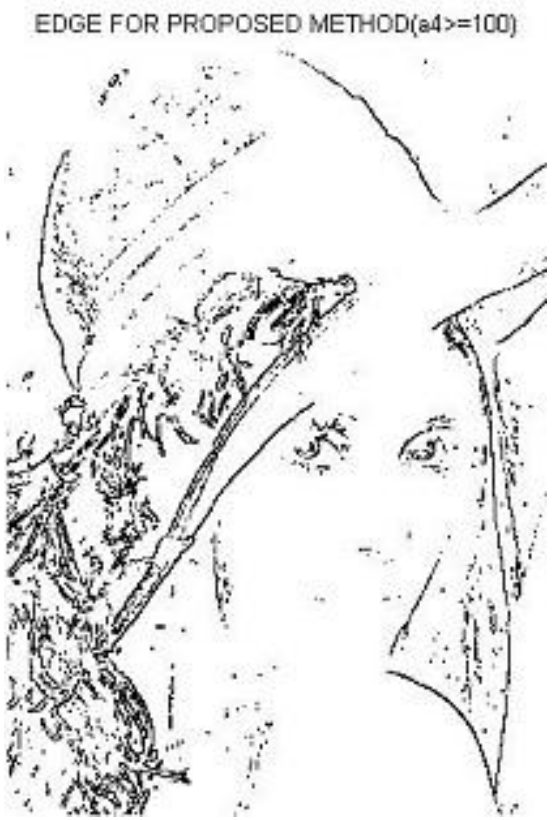

Figure 8(a): Edge for proposed $\operatorname{method}\left(\mathrm{a}_{4}>=100\right)$ EDGE FOR PROPOSED METHOD $(a 4>=80)$

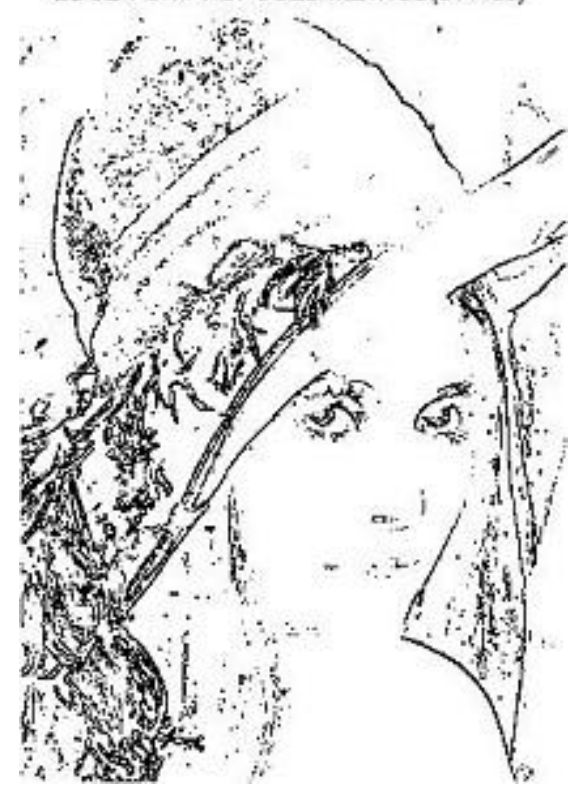

Figure 8(b): Edge for proposed $\operatorname{method}\left(a_{4}>=80\right)$ 


\section{International Journal of Science and Research (IJSR) \\ ISSN (Online): 2319-7064}

Index Copernicus Value (2013): 6.14 | Impact Factor (2014): 5.611

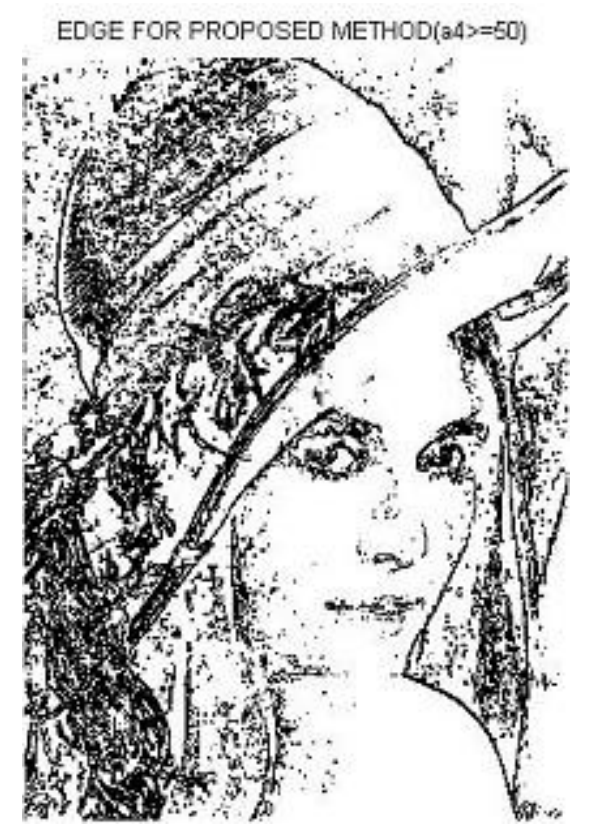

\section{Advantages of the New Method}

This paper proposes a new method for edge detection for the images using MATLAB. To perform Edge detection on gray level image, consider improving the program of Prewitt Operator used for edge detection for the gray level images. The number of instructions and loops in the proposed method is less than Prewitt Operator which reduces the total time of executing program as a result; it reduces the usage of center process unit $\mathrm{CPU}$ to get fast processing for the images which are used in real time signal processing. Table 1 depicts the execution time (Elapsed time) in seconds for each Operator.

Figure 8(c): Edge for proposed method $\left(a_{4}>=50\right)$

Table 1: The execution time (Elapsed time) in seconds for each method

\begin{tabular}{|c|c|c|c|c|c|}
\hline \multirow{2}{*}{ Test images } & \multirow{2}{*}{ Size of image } & \multicolumn{4}{|c|}{ Elapsed Time for each method in (Seconds) } \\
\cline { 3 - 6 } & & Pervert edge & Robert edge & Sob ledge & New method \\
\hline Cameraman image & $256 \times 256$ & 1.847313 & 0.768117 & 1.467503 & 1.028045 \\
\hline Lena image & $420 \times 300$ & 3.296744 & 1.417789 & 2.705492 & 1.510194 \\
\hline
\end{tabular}

\section{Conclusion}

Edge detection is important feature for an image which may be used for image identification. The proposed method is a computationally simpler and more efficient method for computation of edges and proves to be useful in finding edges with much less computations and is simple to implement. It proves to give results that are as good as and even better than Prewitt edge program.

\section{References}

[1] Gonzalez and Woods, 2008. Digital Image Processing, Third Edition, Pearson Education.

[2] Hou, T. H. and Kuo, W.L., 1997. "A New Edge Detection method for Automatic Visual Inspection", International Journal of Advanced Manufacturing Technology, (1997) 13:407412 (C) 1997 Springer-Verlag London Limited.

[3] Jiang, X. and Bunke, H. 1998. "Edge Detection in Range Images based on Scan Line Approximation", Computer Vision and Image Understanding, Vol. 73, No. 2, February, pp. 183-199, 1999 Article ID cviu.1998.0715, available online at http://www.idealibrary.com.

[4] Genming, C. and Bouzong, Y., 1989. "A New Edge Detector with Thinning and Noise resisting Abilities", Journal of Electronics, China, Volume 6, No. 4, 314-319, DOI:10.10071BF02778914.

[5] Qixiang, Ye, Wen, G., Weiqquiang, W. 2003. “A New Texture Insensitive Edge Detection Method",Institute of Computing Technology, Chinese Academy of Sciences,China, ICICS-PCM 2003, 15-18 Dec 2003, Singapore.

[6] Caragea S. 2008. Fondater, Administrator and Chief Editor, IntelliProject, "Difference Edge Detection", Licensed under
IntelliProject open License, Romania, http://www.intelliproject.net.

[7] Rital, S., Bretto A., Cherifi, H., Aboutajdine, D. 2002. "A combinatorial edge detection Algorithm on Noisy images", VIPromCom-2002, 4th EURASIP- IEEE Region 8 International Symposium on Video/image Processing and Multimedia Communications, 16-19 June 2002, Zadar, Croatia.

[8] Fesharaki N. Mehdi, Hellestrand Graham R. 1994. "A New Edge Detection Algorithm based on Statistical Approach", 1994 International Symposium on Speech, Image Processing and Neural Network, 13-16 April, 1994, Hong Kong.

\section{Author Profile}

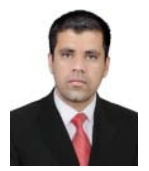

Muthanna Ali Saihood have attained his B.Sc. in Electrical and Electronic Engineering from University of Technology-Baghdad in 2004. From 2006-2013, he had worked in Control and Measurement Department in Nassiryah Thermal Power Station to produce Electrical Energy, Ministry of Electricity. He is currently pursuing master's degree in Electronic and Communication Engineering in College of Engineering \& Technology, Acharya Nagarjuna University, Andhra Pradesh.

Mrs. G. Prathibha has done her B.Tech in R.V.R \& J.C college of Engineering from 2001-2005 and M.Tech in JNTU, Hyderabad from 2005-2007. Her area of interests include Image Processing, Pattern Recognition, Signal processing, Video Processing etc, She is currently working as an Asst Professor in the department of Electronic and Communication Engineering, Acharya Nagarjuna University, Guntur, Andhra Pradesh, India.

\section{Volume 4 Issue 12, December 2015}

